\title{
44598 - LARGE DOSE PROPOFOL DURING ISCHEMIA AND EARLY REPERFUSION INCREASES NITRIC OXIDE BIOAVAILABILITY TO CONFER CARDIOPROTECTION IN ACPB PATIENTS
}

\author{
David Ansley, University of British Columbia Dept of Anesthesia, Vancouver, BC, Canada; \\ Hajieh Ghasemia Safaei, University of British Columbia; \\ Koen Raedschelders, University of British Columbia; \\ Tao Luo, University of British Columbia; \\ Richard Cook, University of British Columbia; \\ A Karim Qayumi, University of British Columbia;
}

Introduction: We tested the hypothesis that large dose propofol confers cardioprotection by increasing tissue nitric oxide bioavailability in patients during coronary surgery with cardiopulmonary bypass $(1,2)$.

Methods: Local IRB approval was obtained for this study. Thirty-two patients scheduled for ACBP surgery were randomized in a double blind study to receive isoflurane 0.5 to $2 \%(n=16)$ or propofol, one $\mathrm{mg} / \mathrm{kg}$ then $120 \mathrm{ug} / \mathrm{kg} / \mathrm{min}(\mathrm{n}=16)$ for a treatment interval of 120 to $150 \mathrm{~min}$ (10 min before and during $\mathrm{CPB}$, and for $15 \mathrm{~min}$ following aortic declamping). Coronary sinus blood and atrial tissue were sampled prior to aortic cross-clamp and at 5-15 min reperfusion. The levels of plasma free 15-F2t- isoprostane and troponin I were determined by ELISA. Cardiac tissue eNOS and iNOS protein and gene expression were measured by Western blot and real time PCR. Hemodynamics were measured prior to and following separation from CPB. Results: Plasma free 15-F2t- isoprostane did not increase significantly during reperfusion in either group. Intraoperative Troponin I increased significantly during reperfusion in the isoflurane group $(\mathrm{P}<0.05)$ and a level greater than $0.5 \mathrm{ng} / \mathrm{ml}$ was associated with myocardial infarction in two patients. There were no significant inter-group differences in postoperative Troponin I up to 24 hours.

The eNOS protein expression and mRNA levels in pre- and post bypass atrial tissue did not change in patients treated with isoflurane $(\mathrm{P}>0.05)$, but increased significantly post bypass in the propofol group $(\mathrm{P}<0.05)$. This was associated with upregulation of iNOS gene expression compared to isoflurane. Overall, iNOS gene expression was similar in isoflurane and propofol groups $(\mathrm{P}>0.05)$. iNOS protein expression was undetectable in pre and post bypass biopsies taken from patients in either anesthetic group .

Cardiac Index was significantly greater for the first two hours post bypass in the propofol group compared with isoflurane treatment $(\mathrm{P}<0.05)$.

Conclusion: Large dose propofol applied during cardiopulmonary bypass significantly decreases Troponin I release during reperfusion and upregulates myocardial eNOS protein and gene expression. We conclude propofol increases myocardial nitric oxide bioavailability to confer cardioprotection in ACBP patients(3). The mechanisms have yet to be elucidated. The pattern of gene expression for eNOS/iNOS is associated with enhanced cardiac performance and suggests the possibility that propofol may mediate delayed preconditioning of the myocardium. 
Further investigation is required to determine whether this reflects the action of propofol alone, or propofol acting in synergism with isoflurane. We provide mechanistic support for the use of propofol as a preemptive cardioprotectant in select populations where the $\mathrm{mK}+$ ATP channel is corrupted.

References

1.Jones , Bolli. J Mol Cell Card 2006; 40: 16-23.

2.Cohen, et al. Cardiova Res 2006; 70: 231-239.

3.Baker, et al. Cardiova Res 1999;43: 685-697. 